\title{
Single-photon cooling in a wedge billiard
}

\author{
S. Choi ${ }^{1}$, B. Sundaram ${ }^{1}$, M. G. Raizen ${ }^{2}$ \\ 1 Department of Physics, University of Massachusetts, Boston, MA 02125, USA \\ 2 Department of Physics, The University of Texas, Austin, Texas 78712, USA
}

\begin{abstract}
Single-Photon Cooling (SPC), noted for its potential as a versatile method for cooling a variety of atomic species, has recently been demonstrated experimentally. In this paper, we study possible ways to improve the performance of SPC by applying it to atoms trapped inside a wedge billiard. The main feature of wedge billiard for atoms, also experimentally realized recently, is that the nature of atomic trajectories within it changes from stable periodic orbit to random chaotic motion with the change in wedge angle. We find that a high cooling efficiency is possible in this system with a relatively weak dependence on the wedge angle, and that chaotic dynamics, rather than regular orbit, is more desirable for enhancing the performance of SPC.
\end{abstract}

PACS numbers:

\section{INTRODUCTION}

Single-Photon Cooling (SPC) is a general cooling method applicable to most of the Periodic Table as well as molecules [1]. Based on one-way wall of light [2 44], it relies on irreversible optical pumping with one photon scattering to trap atoms inside an optical dipole trap. The irreversibility is achieved by exciting, with a depopulation beam, magnetically trapped atoms to an intermediate hyperfine level from which they decay into an optically trappable state with a finite probability. This process accumulates atoms inside an optical dipole trap without the need for a cycling transition. The key mechanism behind SPC is that only those atoms with kinetic energy less than a threshold energy are captured by the optical trap i.e. cooling is achieved by "filtering" and then isolating colder atoms inside an optical trap. The optical dipole trap is constructed in the form of a box in SPC experiments and hence is simply referred to as the "box" [1].

The nature of SPC implies that a high cooling efficiency can be achieved if as many atoms as possible can be made to encounter the one-way wall of the box with the right kinetic energy. However, various practical constraints exist to limit the cooling efficiency. For instance, it is not productive to move the box around and stir the atomic cloud with a depopulation beam as this can result in significant loss of atoms - there is a high probability that the atoms encountering the box do not have the right energy to be trapped. The box is therefore assumed to be stationary throughout the evolution. The finite size of the box means many of the atoms simply will not pass through the position where the box is placed. The usual isotropic trap is not necessarily the best potential for SPC, since the atomic trajectories within an isotropic trap are evenly spread rather than localized near a region where the box can be placed for efficient collection of the atoms. The energy consideration means that even if the trajectories of the atoms are densely concentrated near one region it is of no use if the atoms are moving too energetically to be trapped.

In order to find the best conditions for SPC, we con- sider in this paper a special system in which various types of atomic trajectories can be demonstrated: the wedge billiard for atoms. Wedge billiard for atoms is a realization of the symmetric gravitational wedge originally introduced by Letihet and Miller [5] which, owing to the singularity of the vertex, demonstrates amazingly rich physics - the atomic trajectories can be tuned from stability to chaos with the change in wedge angle. The wedge billiard for atoms has already been implemented experimentally [6] where the atomic motion in different regimes of classical chaos were observed and compared with numerical simulations. In this paper, we consider SPC in wedge billiard and, more specifically, look for the best position to place the box for all possible wedge angles. The best box position is defined as the position at which the most number of atoms is captured.

The overall goal of a cooling scheme is to increase the phase space density. Phase space density in the context of cooling is defined as the number of atoms in a box with sides of one thermal de Broglie wavelength [7]. This can be written as $\rho=(N / V) \lambda_{d B}^{3}$ where $N$ is the number of atoms, $V$ the volume, and $\lambda_{d B}$ is the thermal de Broglie wavelength $\lambda_{d B}=h / \sqrt{2 \pi m k_{B} T}$ where $h$ is the Planck constant. This implies that the factor by which the phase space density changes is given by

$$
\frac{\rho_{f}}{\rho_{i}}=\frac{N_{b o x}}{N_{i}} \frac{A_{\text {wedge }}(\theta)}{A_{\text {box }}}\left(\frac{T_{i}}{T_{f}}\right)^{3 / 2}
$$

where $A_{\text {wedge }}$ and $A_{b o x}$ are the areas of the 2-dimensional wedge and the box, and $T_{i}$ and $T_{f}$ denote respectively the initial and final temperature. The fraction of atoms captured by the box, $N_{\text {box }} / N_{i}$ is therefore an important measure, although it has less impact than the ratio of the final to initial temperature which is raised to the power of $3 / 2$. We shall define in this paper the logarithm of Eq. (1) as the cooling efficiency

$$
\eta=\log _{10}\left(\rho_{f} / \rho_{i}\right) .
$$

We are most interested in how the atoms can be made to attain the right energy and how the cooling efficiency is affected by the regular and chaotic dynamics; from this, 
one may gain understanding of how best to implement the SPC in general.

The paper is organized as follows: In Section II we discuss how the numerical simulation is carried out, including the parameters used and the three types of boxes we use in our investigation. In particular, we discuss how the results may be generalized, and identify the core parameter space for this system, namely the wedge angle, the initial temperature of the atoms and the box threshold energy. In Section III, we present the numerical results for the case that corresponds to the actual experimental parameters, and lay the foundation for the subsequent section by showing how various quantities such as the fraction of trapped atoms change for different box types and wedge angles. In Section IV we present the result of simulations that cover various combination of the three core parameters to establish the general trend regarding the cooling efficiency of SPC in a wedge billiard for atoms. We conclude in Section V.

\section{SIMULATION METHOD}

\section{A. Atomic trajectories in wedge billiard}

First, we assume that atomic wedge billiard contains thermal atoms. The initial positions and momenta of the atoms are assigned according to a Gaussian random distribution as is usually done for thermal atoms in equilibrium. In particular, the initial momenta of the atoms are determined from the Maxwell-Boltzmann distribution $f\left(v_{x}, v_{y}\right)=\left(\frac{m}{2 \pi k_{B} T}\right)^{3 / 2} \exp \left[-\frac{m\left(v_{x}^{2}+v_{y}^{2}\right)}{2 k_{B} T}\right]$ such that the variance of velocity $\sigma^{2}=k_{B} T / m$ is proportional to the the initial temperature of the atomic cloud, $T$. Here $k_{B}$ is the Boltzmann constant and $m$ is the mass of the atom. Assuming hard walls for the wedge billiard such that atoms undergo elastic collisions with the walls and taking the scattering cross section for the interatomic collision to be zero, we calculate the expected trajectory of each atom within the wedge billiard using classical kinematic equations as done in Ref. [6]. The calculated trajectory for each of the atoms is stored and used later for the analysis involving the box.

The number of atoms in the sample, $N$, is typically be of the order $\sim 10^{6}$ experimentally but in order to simulate a realistic system using a computationally manageable number of atoms we take $N$ to be a few hundred and average the final result over several runs with different initial position and velocity (but with the same velocity variance i.e. the same initial temperature). With 200 atoms in each run, averaging over 50 runs gave results similar to a single run with 10000 atoms. For each run, the $N$ trajectories corresponding to that particular set of initial conditions were used to calculate the fraction of atoms trapped and lost by each type of the box discussed below. These fractions are later averaged over the number of runs to give a better estimate for a realistic atomic sample. The averaging has the effect of smoothing out any large fluctuations in the result; in fact, since we have a conservative, closed system, the fraction of atoms captured by the box was found to be fairly consistent for all $N$.

It was also found from our simulations that a significant proportion of atoms bounce off the walls and escape the wedge billiard, especially in the chaotic regime; such atoms obviously cannot be trapped by the box and are considered lost from the system. In an attempt to avoid such loss and to perhaps improve the performance, we have tried modulating the wedge angle during the time evolution but found that it didn't lead to any better result. Such a set up is also likely to be difficult to implement experimentally. We therefore consider in our simulations wedge billiard with the walls fixed at one wedge angle at a time throughout the entire duration of the experiment.

\section{B. Modeling the box}

Numerically, the box is simply modeled as a region in space with a certain implicit threshold energy $E_{b o x}$. The kinetic energy of individual atoms varies greatly throughout the evolution, with the majority of the atoms taking on kinetic energies far exceeding $E_{b o x}$. For a typical $i$ th atom with initial position $\left(x_{i}, y_{i}\right)$, initial velocity $\left(v_{x}^{i}, v_{y}^{i}\right)$, and initial total energy $E_{i}=\frac{1}{2} m\left(v_{x}^{i 2}+v_{y}^{i 2}\right)+m g y_{i}$ where $g$ denotes gravitational acceleration, there are times $t_{k}$, $k=1,2,3 \ldots$ during its evolution where the atom reaches a certain height $y$ that overlaps with the position of the box such that $E_{i}-m g y \leq E_{b o x}$. At these times, the atom may be captured by the box. However not all atoms are captured in reality: based on Ref. [1], the efficiency of the box is typically $\sim 85 \%$ i.e. only $85 \%$ of the atoms that pass through the box "sees" the box (converted to the right hyperfine state). This means $85 \%$ of the atoms that pass through the box are either lost (too high energy) or trapped (correct energy) and 15\% just passes through. We include this constraint in our simulations. It is noted that since the atoms that pass through the box with kinetic energy between zero and $E_{b o x}$ are assumed confined by the box, the final equilibrium temperature of the atoms inside the box can be estimated to be of the order $E_{b o x} / 2 k_{B}$. Finding the right position to place a box given that there are $N$ atoms with different trajectories is a particularly difficult optimization problem, and is the main goal of this paper. To solve the problem of where to place our box 3 types of boxes were considered in our simulation: The optimum box and two types of fixed box we refer to as Type I and Type II box. We explain these in more detail below. 


\section{Optimum box}

Since we are considering a deterministic system, it is, in principle, possible to calculate precisely where and when each atom reaches the required kinetic energy for it to be trapped. The optimum box is a hypothetical box of vanishing size that, by methodically moving around the trap, captures all theoretically trappable atoms in sequence. This then gives the upper limit to the number of atoms that can be captured for a given set of parameters. To provide a more useful theoretical upper limit to the number of trappable atoms, we additionally impose a couple of practical constraints. One of these constraints is that the optimum box is assumed not able to be at more than one position at one given instant - if there are more than one atom attaining trappable energy at the same time only one of them is considered captured and the rest are let evolving. The optimum box subsequently catches the next available atom that reaches the correct energy, and so on. The other constraint is, in line with the real experiment, we assume the efficiency of the optimum box to be $85 \%$. i.e. only $85 \%$ of the atoms passing through the optimum box are captured (all $85 \%$ are captured since, by definition, all atoms intercept the optimum box with the correct kinetic energy).

\section{Type I and Type II (fixed) box}

In reality, the box is finite in size and cannot be moved around freely. We consider two possible cases in relation to where to place the fixed box. One is to use the result of the optimum box calculation above to guide us where to place our box. Based on the optimum box calculation which gives a sequence of box positions over the duration of the experiment, we choose to place a real, finitesized, stationary box at the position where the largest number of the calculated trappable positions fall within the width of box, in both $x$ and $y$ directions. We call this stationary box Type I box. On the other hand, in the absence of an optimum box calculation, the best one can do is to actually place the finite fixed box at various different places inside the wedge billiard to find, by trial-and-error, the position where the most atoms can be trapped. Given the symmetry of the wedge billiard system around $x=0$, after collecting results corresponding to all possible heights of the box on the axis of symmetry, we found the one height $y$ that traps the most atoms for a given wedge angle. We refer to this box obtained from "optimization by hand" as Type II box.

\section{Parameters used}

Using realistic parameters is obviously crucial for the correct modeling of the system being simulated. With too big or too small dimensions, the atomic trajectories and hence the calculated cooling efficiency is likely to be unrealistic. To address this issue we base our parameters on the experimental values [1, [6] and choose our parameters to be within reasonable range of these values. In addition to simulating a realistic system, one should ideally be able to generalize the result beyond the existing experiment. However it is noted that, especially in the chaotic regime, it is impossible to write down analytically a general expression of the atomic trajectories as a function of various parameters. This limits our options to only those of numerical analysis. Since one cannot cover every single possible value of various parameters, only a general trend can be identified from a numerical study. Such general trend should, however, provide sufficient information to understand the fundamental physics of the system, and give us a clue as to the range of realistic cooling efficiencies possible with this system.

The interdependence of various parameters means the parameter space boils down to three independent variables: the wedge angle, initial temperature, and the box threshold energy. It is noted first of all that the regular and chaotic behavior exhibited by the atoms inside the wedge billiard is dependent only on the wedge angle, and is independent of factors such as the atomic species, initial temperature, and the qualities of the box. We therefore capture all the necessary physics in our simulation by scanning through all possible wedge angles. Also, in our numerical model, different atomic species is represented only via their different atomic mass which shows up in the velocity variance $\sigma^{2}$ and in the calculation of the kinetic energy $E_{k}=\frac{1}{2} m v^{2}$. It is noted that any difference in physics due to different atomic mass is taken care of with an appropriate change in the initial temperature $T_{i}\left(\sigma^{2} \propto T_{i}\right)$, and the kinetic energy $E_{k}$ is automatically scaled accordingly. This can be shown by setting $k_{B}=T_{i}=m=1$ so that the initial thermal energy $E=k_{B} T_{i}=1$ becomes unit energy and $\sigma=\sqrt{k_{B} T_{i} / m}=1$ becomes unit velocity such that the unit length $l$ can be defined as $l=\sigma \tau$ where $\tau$ is unit time. The kinetic energy of an atom traveling with velocity $v$ is then given in the scaled unit as $E_{k}=\frac{1}{2}(v / \sigma)^{2}$ i.e. the kinetic energy relative to the initial thermal energy characterized by $T_{i}$ is what matters. All the major physics of this system can therefore be covered by studying various combination of wedge angle, initial temperatures and the box threshold energies.

\section{RESULTS FOR SEVERAL WEDGE ANGLES}

In this section, we illustrate the physics of the system by presenting the results of numerical simulation for select wedge angles using the experimentally verified parameters of Refs. [1] and [6]. The results of our simulation for various regimes characterized by the three different wedge angles $30^{\circ}, 50^{\circ}$, and $80^{\circ}$ are presented in Figs. [1]3 respectively. In each of these figures, we present several sub-figures. First of all, as one of the sub-figures, we present the Poincaré section for the wedge 


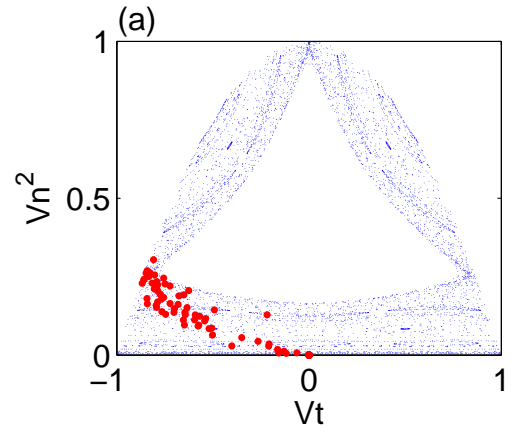

(c)

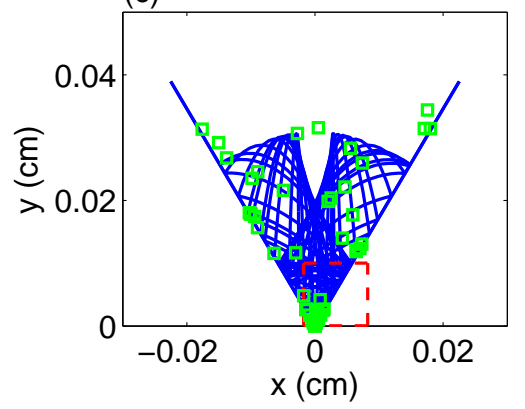

(b)

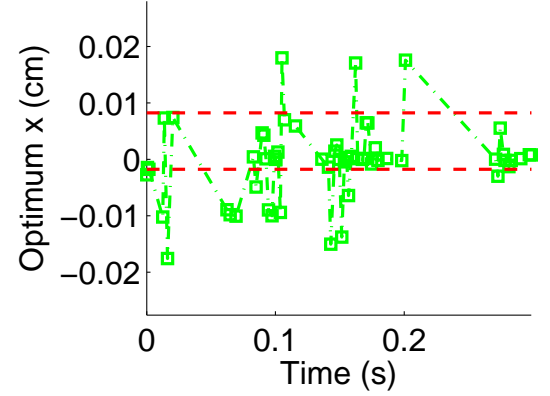

(d)

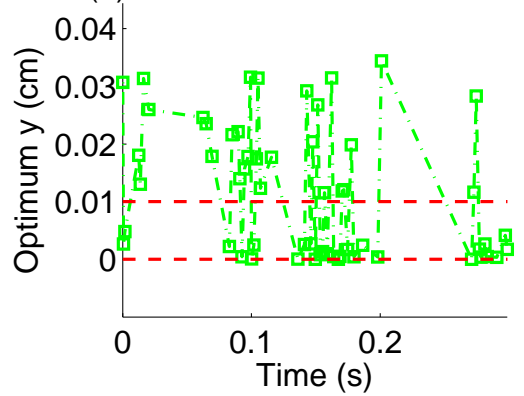

FIG. 1: (Color online) (a) Poincaré section, $v_{t}$ vs. $v_{n}^{2}$ for the wedge billiard with wedge angle $\theta=30^{\circ}$ (smaller blue dots). The larger red dots represent the distribution of the thermal atoms after their first bounce on the wedge walls. The right hand column, i.e. panels (b) and (d), show the $x$ and $y$ component of the optimum box position over time as green squares. The dash-dot line connects the green squares in sequence to show how the optimum box position changes over time. The red dashed lines mark the edges of the Type I box. (c) The wedge in real space with one typical trajectory for an atom shown as blue line. The Type I box used in our simulation is shown in the same panel as a red box in dashed line. The green boxes mark the (changing) positions of the optimum box over the duration of the simulation.

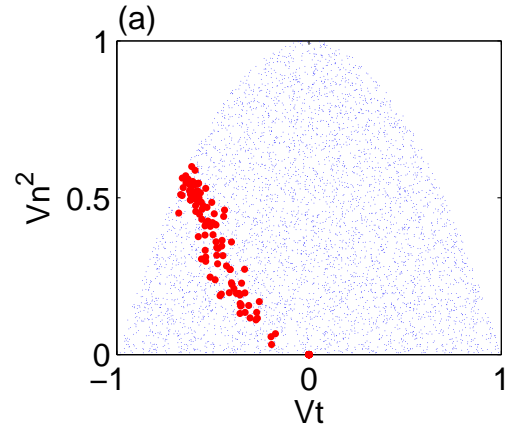

(c)

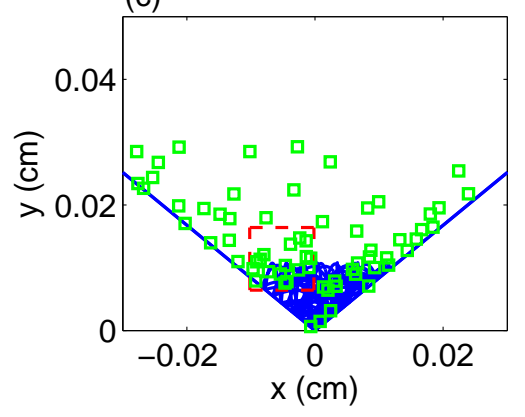

(b)

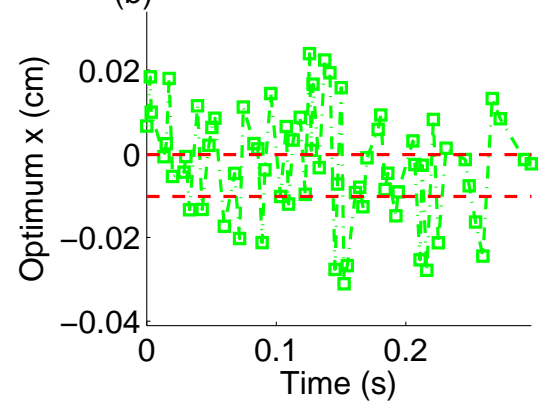

(d)

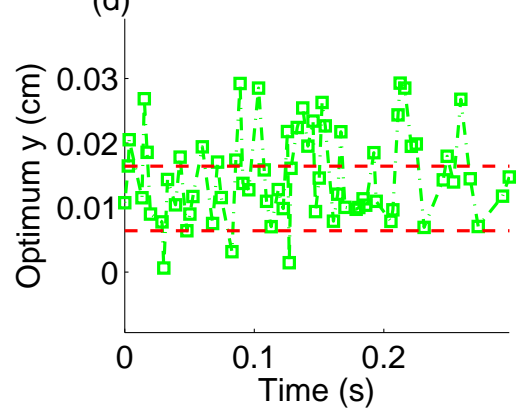

FIG. 2: (Color online) Same as Fig. 1 except the wedge angle is now $50^{\circ}$. 


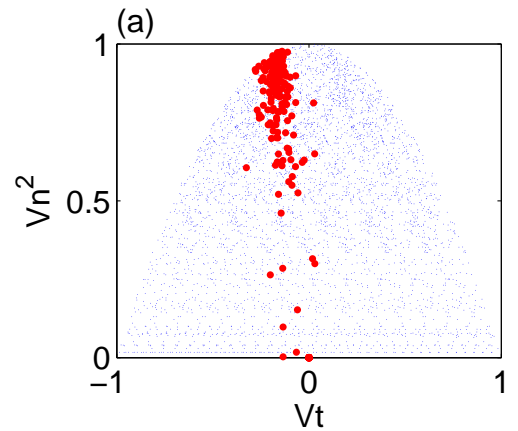

(c)

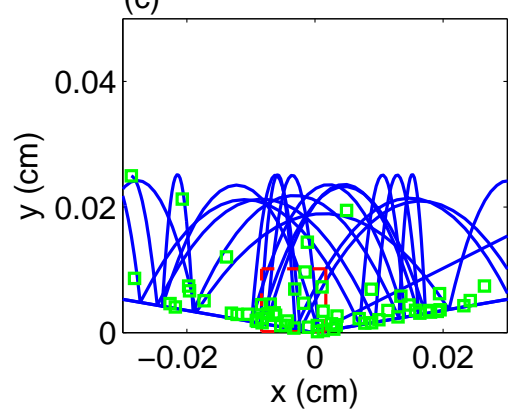

(b)

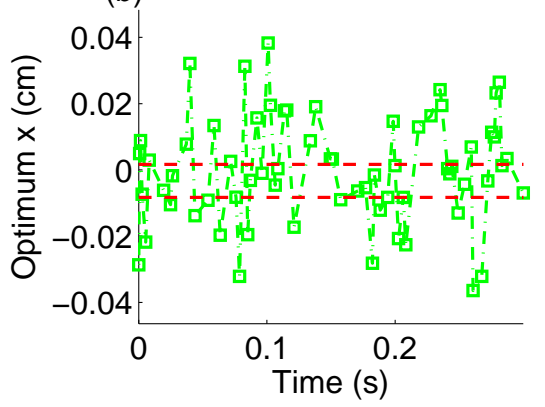

(d)

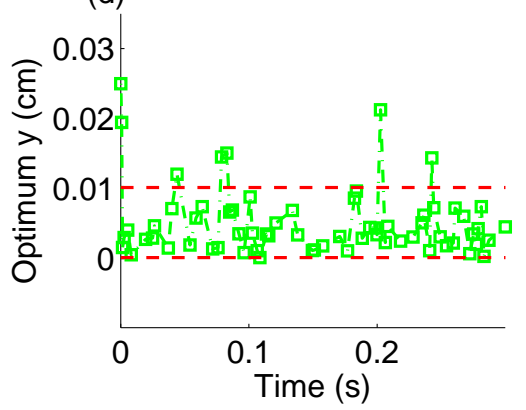

FIG. 3: (Color online) Same as Fig. 1 except the wedge angle is now $80^{\circ}$.

billiard typically presented as a plot of $v_{t}$ vs. $v_{n}^{2}$ where $v_{t}$ and $v_{n}$ denote transverse and normal velocity of the atoms immediately after hitting the walls. As is well known, visible geometric structures or "islands" within the Poincaré section represent regions of regular orbit while the regions containing evenly spread dots correspond to chaotic dynamics. The fact that there are no islands for wedge angles $50^{\circ}$ and $80^{\circ}$ (Figs. 22 and 3) means that all atoms are expected to undergo chaotic dynamics, while the existence of an island for wedge angle $30^{\circ}$ (Fig. 1) means atoms with initial conditions that lie in the island undergo regular orbits. The initial thermal velocity distribution in terms of $v_{t}$ and $v_{n}^{2}$ after one bounce off the walls are shown as (thicker) red dots on the Poincare section as a guide to the initial condition used. Next, typical trajectory of one particular atom in the wedge billiard is presented as well as all the positions of the optimum box in small green squares. The outline of the Type I box determined from the optimum box calculation is also shown superposed in the same sub-figure. In the right hand column of the figures, the time-varying positions of the optimum box decomposed into $x$ and $y$ components are presented along with the boundaries of the Type I box shown in dashed lines.

In Fig. 4 we present various fractions as a function of wedge angles - atoms trapped by the box, atoms remaining in the wedge, and atoms lost for all 3 box types. As mentioned previously, there are two mechanisms by which the atoms are lost - one by encountering the box with the wrong energy and the other by escaping the wedge billiard after the collision with one of the walls. We also show the $x$ and $y$ components of the Type I and Type II boxes as a function of wedge angle. It is found that the Type II box gives slightly better result in terms of the fraction of atoms trapped. It is seen that, for the parameters used, from $5 \%$ to up to around $15 \%$ of the atoms could be trapped by this scheme. The fraction of atoms trapped has (discounting fluctuations) almost linear dependence on the wedge angle with the largest fraction of atoms trapped at $\theta=80^{\circ}$. These observations imply that chaotic dynamics, rather than the regular dynamics, is more conducive for SPC. This makes sense since, to be trapped by the box, the atom has to reach the correct height relative to the initial energy that corresponds to the right kinetic energy. This is more likely in the regime of chaotic dynamics in which the atoms take on various different trajectories over time rather than that of regular orbit which generally has higher kinetic energy and a limited range of trajectories. The wide range of trajectories also means that more atoms are likely to encounter the stationary box. The higher fraction of atoms trapped for larger wedge angle can be explained from the fact that with wider wedge angles, the normal velocity component $v_{n}$ dominates that of the transverse velocity component $v_{t}$ and hence one has a higher fraction of atoms in parabolic motion. With parabolic motion it is easier for the atoms to attain the trappable energy as the kinetic energy goes right down to zero at the turning points.

Finally we present here a simple analysis regarding the height of the best fixed box by assuming that the box is able to catch the atom after one bounce from the walls. Taking the cusp of the wedge billiard as the origin of 

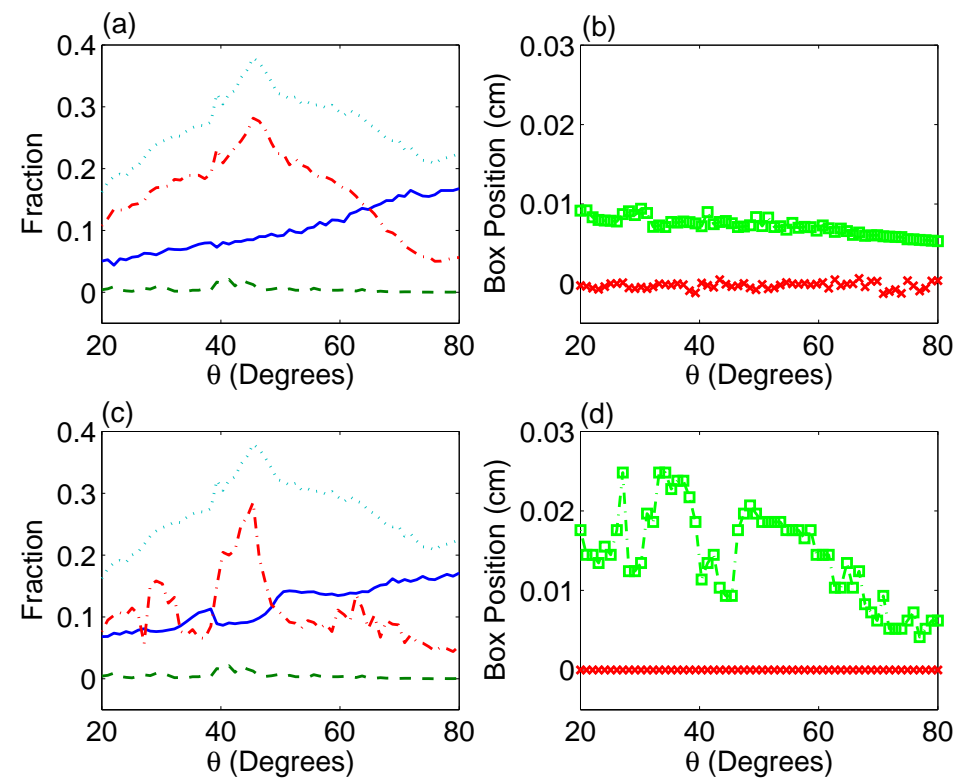

FIG. 4: (Color online) Panel (a) displays the final (after time $t=300 \mathrm{~ms}$ ) fraction of trapped atoms (Solid line), atoms remaining in the wedge (Dashed line), and atoms lost (Dash-dot line) for various wedge angles $\theta \in\left[20^{\circ}, 80^{\circ}\right]$ for Type I box. The additional dotted line represent the fraction of trapped atoms for the case of optimum box, i.e. the theoretical upper limit for comparison (b) The Type I box position for different wedge angles - the squares represent the $y$ component of the mean position and the crosses mark the $x$ component. (c) and (d): Same as (a) and (b) but for Type II box.

position, and considering an atom initially at position $x_{i}$ and $y_{i}$ with initial velocity components $v_{x}^{i}$ and $v_{y}^{i}$, the $x$ and $y$ components of the velocity on impact with the wall at angle $\theta$ from the perpendicular line at the origin are $v_{x}^{w}=v_{x}^{i}$ and $v_{y}^{w}=\sqrt{v_{y}^{i 2}-2 g\left[\left|y_{i}\right|-\left|x_{i}\right| \tan \left(\frac{\pi}{2}-\theta\right)\right]}$. Energy conservation implies:

$$
m g h=\frac{1}{2} m\left(v_{x}^{w 2}+v_{y}^{w 2}\right)-E_{b o x},
$$

where $E_{b o x}$ is the threshold energy of the box. This gives the best box height as

$$
\begin{aligned}
h & =\frac{E_{K}^{i}-E_{b o x}}{m g}-\left|y_{i}\right|+\left|x_{i}\right| \tan \left(\frac{\pi}{2}-\theta\right) \\
& \approx \frac{E_{K}^{i}-E_{b o x}}{m g}-\left|y_{i}\right|+\left|x_{i}\right|\left(\frac{1}{\theta}-\frac{\theta}{3}-\cdots\right) .
\end{aligned}
$$

This result is valid for larger $\theta$ where the first bounce results in a parabolic trajectory e.g. when $y_{0}>L_{w} \cos \theta$ where $L_{w}$ is the length of the wedge wall. For smaller $\theta<50^{\circ}$ a regular orbit is likely which means any analysis based on one bounce does not hold. A plot of $h$ given by Eq. (4) was found to closely reproduce the large angle $\theta>50^{\circ}$ part of Fig. 4 (d).

The cases where $v_{x}$ is large enough (or $\theta$ is small enough) so that the atom hits the wall due to the horizontal rather than the vertical component of motion require a modified analysis: Assuming the time it takes for an atom to hit the wall is $\tau_{x}$, the $y$ component of the velocity at the wall is $v_{y}^{w}=v_{y}^{i}-g \tau_{x}$ while $x$ component of the velocity at the wall is $v_{x}^{i}$. The vertical displacement after $\tau_{x}$ is $y_{i}-\frac{1}{2} g \tau_{x}^{2}$ and this means the distance traversed horizontally $v_{x}^{i} \tau_{x}=\left[y_{i}-\frac{1}{2} g \tau_{x}^{2}\right] \tan \theta$, i.e. $g \tau_{x}=\cot \theta\left[-v_{x}^{i}+\sqrt{v_{x}^{i 2}+2 g y_{i} \tan ^{2} \theta}\right]$. The energy conservation then implies

$$
\begin{aligned}
h= & \frac{E_{K}^{i}-E_{b o x}}{m g}-v_{y}^{i} \tau_{x}+\frac{1}{2} g \tau_{x}^{2} \\
= & \frac{E_{K}^{i}-E_{b o x}}{m g}+y_{i}-\frac{\cot \theta}{g}\left(v_{y}^{i}+v_{x}^{i} \cot \theta\right) \\
& \times\left[\sqrt{v_{x}^{i 2}+2 g y_{i} \tan ^{2} \theta}-v_{x}^{i}\right] \\
\approx & \frac{E_{K}^{i}-E_{b o x}}{m g}-\frac{y_{i} v_{y}^{i}}{v_{x}^{i}} \theta+\frac{g y_{i}^{2}}{2 v_{x}^{i 2}} \theta^{2}+\cdots
\end{aligned}
$$

This reproduces the result for smaller $\theta$ but again, since we are only considering one bounce and ignoring the possibility of a regular orbit, the oscillatory behavior is not recovered in this very approximate result.

\section{COOLING EFFICIENCY OF SPC IN WEDGE BILLIARD}

In this section, we calculate the cooling efficiency $\eta$ for SPC in wedge billiard. In particular, we identify general features of this system by considering various combination of the three core parameters: wedge angle, initial 
temperature, and the box threshold energy. To give a better sense of the range of parameter values used in this section, we denote the initial temperature and the box threshold energy of existing experiments as $T_{i}^{e x}$ and $T_{b}^{e x}$ respectively and refer to all the other initial temperatures and threshold energies as some multiples of $T_{i}^{e x}$ and $T_{b}^{e x}$.

\section{A. Changing the initial temperature}

In Fig. 5, we present the cooling efficiency for Type I and Type II boxes. Figure 5 is arranged similarly to Fig. 4, except that we present the cooling efficiency $\eta$ for the four different initial temperatures of $T_{i}^{e x}, 10 T_{i}^{e x}$, $50 T_{i}^{e x}$, and $100 T_{i}^{e x}$ for the box threshold temperature of $T_{b}^{e x}$. We also show, as done in Fig. 4, the positions of Type I and Type II boxes as a function of wedge angle for different initial temperatures. We chose to look at such widely varying initial temperatures up to $100 T_{i}^{e x}$ since it was found numerically that smaller changes did not noticeably affect the cooling efficiency e.g. the difference in result between say $T_{i}^{e x}$ and even $4 T_{i}^{e x}$ was not noticeable. Such immunity to temperature changes means the results shown here for each of these four initial temperatures should hold for a wide range of atomic species and length scales. It is seen that there is, in fact, quite a dramatic increase in phase space density - the higher the initial temperature the more significant is the improvement.

It is also seen that due to the interplay of various factors in the calculation of $\eta$, the curves do not show very strong dependence on the wedge angle, although one can notice slight changes in the (shallow) maximum of the curves. The curves have maximum near $70^{\circ}$ for the initial temperature of $T_{i}^{e x}$ and near $50^{\circ}$ for the initial temperature of $100 T_{i}^{e x}$. Given that the ratio of the area $A_{\text {wedge }}(\theta) / A_{\text {box }}$ has maximum at the wedge angle $\theta=45^{\circ}$ this implies that the fraction of atoms trapped (i.e. instead of $\eta$ that also compares the temperature difference) does not change much with the change in the wedge angle for higher initial temperature. Indeed, the fraction of atoms trapped was found to increase almost linearly with the increasing wedge angle, but the gradient of this linear variation was highest for the initial temperature of $T_{i}^{e x}$ and the smallest for the initial temperature of $100 T_{i}^{e x}$. The fact that the fraction of atoms trapped was the highest for $\theta=80^{\circ}$ for all initial temperatures (albeit with a varying degree) re-confirms the main mechanism by which the atoms get trapped by the box - with wider wedge angle, one has a higher fraction of atoms in parabolic trajectory.

The change in phase space density corresponding to the change in initial temperature from $50 T_{i}^{e x}$ to $100 T_{i}^{e x}$ is smaller compared to that with change in initial temperature from $10 T_{i}^{e x}$ to $50 T_{i}^{e x}$ i.e. there is a saturation in the amount of increase in phase space density. This can be partly explained by examining the fraction of atoms trapped by the box. It was found that the fraction of atoms trapped did not change much at all between the initial temperatures of $T_{i}^{e x}$ and $10 T_{i}^{e x}$ i.e. the temperature ratio has a major effect. Over $10 T_{i}^{e x}$, however, the fraction of trapped atoms began to be noticeably reduced. This is because after the temperature gets high enough atoms have enough energy to escape the wedge in significant numbers. With many atoms escaping and reducing the pool of atoms inside the wedge, the actual fraction of atoms trapped by the box goes down, offsetting the effect of the larger temperature ratio.

Finally we note that the results of Type I and Type II are very similar. This is because of two reasons - one is that the maximum fraction of atoms trapped using Type II boxes, while greater than that using Type I box, is not significantly different. The second reason is that the finite box size implies there is actually a significant overlap between the two different types of box positions. The finite box size also means that the slightly different box positions for different initial temperature (for both Type I and Type II) is not noticeable - the temperaturedependent difference in the position of the box center is smaller than the width of the box itself.

\section{B. Changing the box threshold energy}

Similarly to Fig. [5, we show in Fig. 6] the cooling efficiency $\eta$ and the corresponding positions for Type I and Type II boxes. But this time, we fix the initial temperature at $10 T_{i}^{e x}$ and show the general trend with respect to four different box threshold temperatures of $T_{b}^{e x}, 2 T_{b}^{e x}$, $3 T_{b}^{e x}$, and $10 T_{b}^{e x}$. The initial temperature of $10 T_{i}^{e x}$ was chosen to make sure that even with box threshold temperature of $10 T_{b}^{e x}$ the atomic sample is actually being cooled down. The observed trend with increasing box threshold energies is the opposite of the trend observed above with increasing temperature: $\eta$ decreases with increasing box threshold energy, which is unexpected since with increasing box threshold energy, the actual fraction of atoms trapped is increased as the atoms are more likely to be trapped. This can be understood from the fact that, with increasing box threshold energy, the ratio of initial to final temperature is decreased and the temperature ratio is the more significant contributor to $\eta$.

Again, there is a relatively weak dependence of $\eta$ on the wedge angle. The wedge angle at which the maximum $\eta$ occurs is observed to be slightly shifted from near $70^{\circ}$ for the box threshold energy of $T_{b}^{e x}$ to near $50^{\circ}$ for the box threshold energy of $10 T_{b}^{e x}$. As before, this can be explained by the considering the ratio of the areas $A_{\text {wedge }}(\theta) / A_{b o x}$ and the fraction of atoms trapped. In fact, the fraction of the atoms trapped as a function of wedge angle demonstrates quite a different behavior from that seen above for different initial temperatures. Although the fraction of atoms trapped with box threshold energy of $T_{b}^{e x}$ has roughly linear dependence on the wedge angle (with maximum near $80^{\circ}$ ), with higher box threshold energies, the fraction of atoms trapped grad- 

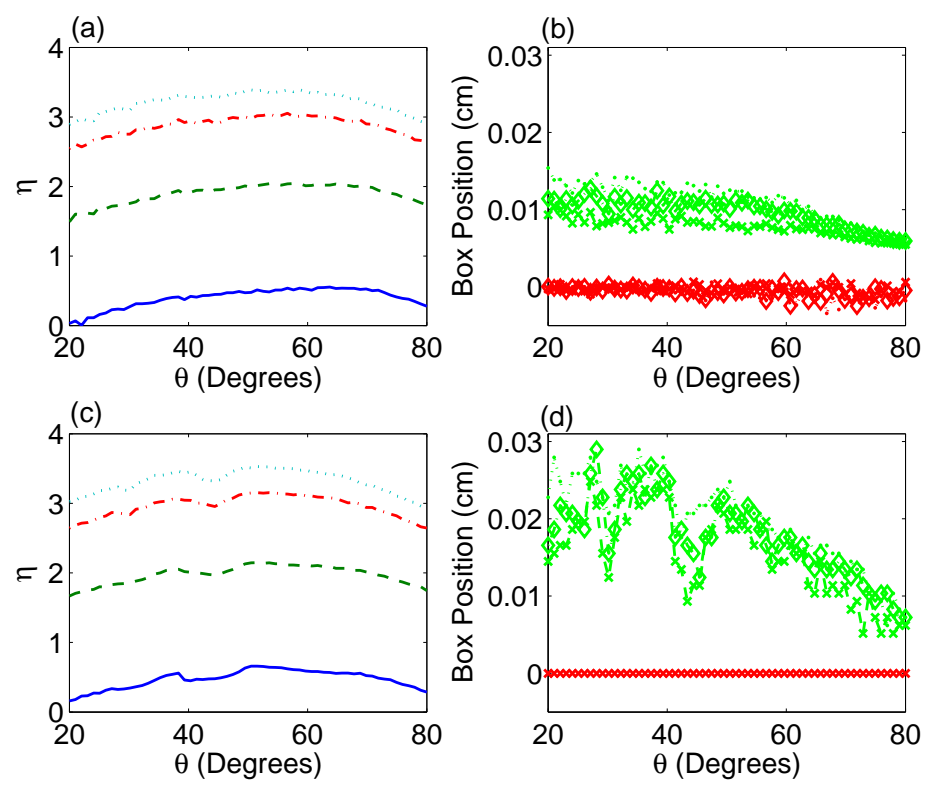

FIG. 5: (Color online) Panel (a) displays the cooling efficiency $\eta=\log _{10}\left(\rho_{f} / \rho_{i}\right)$ for different initial temperatures of $T_{i}^{e x}$ (Solid line), $10 T_{i}^{e x}$ (Dashed line), 50T $T_{i}^{e x}$ (Dash-dot line) and $100 T_{i}^{e x}$ (Dotted line) as a function of wedge angles $\theta \in\left[20^{\circ}, 80^{\circ}\right]$ for Type I box. The box threshold energy is $T_{b}^{e x}$. (b) Type I box position for different wedge angles - the crosses, diamonds, and dots represent the $y$ component of the mean position for the initial temperatures $10 T_{i}^{e x}$ (Dashed line), 50T $T_{i}^{e x}$ (Dash-dot line) and $100 T_{i}^{e x}$ (Dotted line) respectively (Green in color in the online version). The corresponding symbols around zero mark the $x$ component. (Red in color in the online version.) (c) and (d): Same as (a) and (b) but for Type II box.
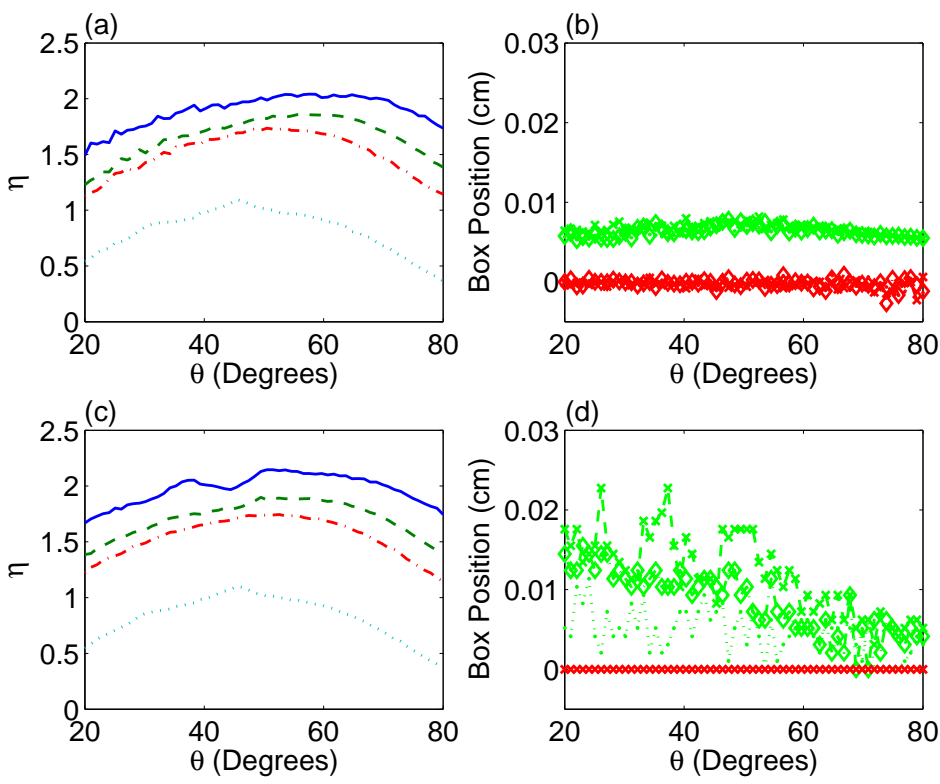

FIG. 6: (Color online) Panel (a) displays the cooling efficiency $\eta=\log _{10}\left(\rho_{f} / \rho_{i}\right)$ for different box threshold temperatures of $T_{b}^{e x}$ (Solid line), $2 T_{b}^{e x}$ (Dashed line), 3T $T_{b}^{e x}$ (Dash-dot line) and $10 T_{b}^{e x}$ (Dotted line) a function of wedge angle $\theta \in\left[20^{\circ}, 80^{\circ}\right]$ for Type I box. The initial temperature was $10 T_{i}^{e x}$. (b) Type I box position for different wedge angles - the crosses, diamonds, and dots represent the $y$ component of the mean position for the box threshold temperatures $2 T_{b}^{e x}$ (Dashed line), $3 T_{b}^{e x}$ (Dash-dot line) and $10 T_{b}^{e x}$ (Dotted line) respectively (Green in color in the online version). The corresponding symbols around zero mark the $x$ component. (Red in color in the online version.) (c) and (d): Same as (a) and (b) but for Type II box. 
ually demonstrates a "triangular" shape as a function of wedge angle (with maximum near $50^{\circ}$ ). Interestingly, with the box threshold energy of $10 T_{b}^{e x}$, it matches the triangular shape seen for the optimum box result as dotted line in Fig. 4(a) and (c). This shows that with large enough box threshold energy, all atoms that can be trapped as calculated by the optimum box are indeed trapped given enough time. This is a significant result since it shows a potential to "simulate" the complicated optimum box calculation experimentally.

A saturation is found to occur as the box threshold energy becomes larger; changes in $\eta$ due to changes in box threshold energy become smaller as the box threshold energy becomes close to $10 T_{b}^{e x}$. This is to be expected since, once the box threshold energy becomes large enough to catch all the atoms that are physically feasible to be caught (i.e. ones that do not escape the wedge billiard), any higher box threshold energy will not give different fraction of atoms trapped. As regards to the actual box position itself and the use of Type I and Type II boxes, similar observation as above is made i.e. the final cooling efficiency for the two different box position is quite similar and that, due to the finite width of the box, difference in box position do not lead to noticeable changes in $\eta$.

\section{CONCLUSION}

We have found that SPC can significantly increase the phase space density of the atoms originally trapped inside a wedge billiard. It was found that even in the very tough scenario of very high initial temperature and very low box threshold energy, enough atoms are expected to be caught to give a high cooling efficiency. The cooling efficiency $\eta$ showed a relatively weak dependence on the wedge angle. This is because $\eta$ depends on the interplay of various factors, not just the number of atoms trapped by the box. For low initial temperature, and also for low box threshold energy, the best angle was near $\theta=70^{\circ}$ and for higher initial temperature and also for higher box threshold energy, the best angle was near $\theta=50^{\circ}$. Various trends could be explained by studying the fraction of atoms trapped as a function of the wedge angle. Both Type I and Type II boxes were found to give similar cooling efficiencies, although the Type II box is one that can be obtained experimentally in the absence of any knowledge about the idealized optimum box calculation.

The regime in which the performance of SPC in wedge billiard is best was identified to be the regime of chaotic dynamics with wedge angle $\theta \geq 45^{\circ}$. In the chaotic regime, atoms take on various different kinetic energies and trajectories, increasing the likelihood of meeting the condition for SPC. Although on first sight atoms in regular orbits look more promising, their kinetic energy is less likely to be redistributed, resulting in a smaller cooling efficiency. Within the chaotic regime, it was found that the wider wedge angle is better in terms of capturing a higher fraction of atoms. This is because, with a wider wedge angle, there are more of atoms undergoing parabolic motion where the kinetic energy becomes zero at the turning point. These observations indicate that, in general, the best set up for SPC is the one where the box is able to access the most number of turning points concentrated within a small region. Somewhat less ideal, but an experimentally more feasible case would be to release the atoms in a rectangular trap [8] i.e. with a flat bottom or on a magnetic mirror [9] so that all the atoms are bouncing in parabolic trajectories, and scan the optical box across so as to "skim" all the trappable atoms from the top, and slowly move down to capture progressively less energetic atoms. With this arrangement the box is always at the top, so there is no worry about unnecessarily stirring atoms using a depopulation beam and losing them. This will be presented in our future work [10].
[1] G. N. Price, S. T. Bannerman, K. Viering, E. Narevicius, and M. G. Raizen Phys. Rev. Lett. 100093004 (2008)

[2] A. Ruschhaupt and J. G. Muga Phys. Rev. A 70, 061604(R) (2004)

[3] M. G. Raizen, A. M. Dudarev, Qian Niu, and N. J. Fisch Phys. Rev. Lett. 94053003 (2005)

[4] J. J. Thorn, E. A. Schoene, T. Li, and D. A. Steck Phys. Rev. A 79, 063402 (2009)

[5] H. E. Letihet, and B. N. Miller Physica (Amsterdam) 21D 93 (1986)

[6] V. Milner, J. L. Hanssen, W. C. Campbell, and M. G.
Raizen Phys. Rev. Lett. 861514 (2001)

[7] C. G. Townsend, N. H. Edwards, C. J. Cooper, K. P. Zetie, C. J. Foot, A. M. Steane, P. Szriftgiser, H. Perrin, and J. Dalibard Phys. Rev. A 52, 1423 (1995)

[8] T. P. Meyrath, F. Schreck, J. L. Hanssen, C.-S. Chuu, and M. G. Raizen Phys. Rev. A 71, 041604(R) (2005)

[9] T. M. Roach, H. Abele, M. G. Boshier, H. L. Grossman, K. P. Zetie, and E. A. Hinds Phys. Rev. Lett. 75, 629 (1995).

[10] S. Choi, B. Sundaram, M. G. Raizen (In preparation) 